\title{
Outcomes of primary versus revisional robotically assisted laparoscopic Roux-en-Y gastric bypass: a multicenter analysis of ten-year experience
}

\author{
Pouya Iranmanesh ${ }^{1}$ (D) John Fam ${ }^{2} \cdot$ Thomas Nguyen $^{1} \cdot$ David Talarico $^{2} \cdot$ Kavita D. Chandwani $^{1} \cdot$ Kulvinder S. Bajwa $^{1}$. \\ Melissa M. Felinski ${ }^{1}$. Leon V. Katz ${ }^{2}$. Sheilendra S. Mehta ${ }^{3}$. Stephan R. Myers ${ }^{2}$ - Brad E. Snyder ${ }^{1}$. Peter A. Walker ${ }^{4}$. \\ Todd D. Wilson ${ }^{1} \cdot$ Angielyn R. Rivera ${ }^{1}$. Connie L. Klein ${ }^{1}$ · Shinil K. Shah ${ }^{1,5} \cdot$ Erik B. Wilson $^{1}$
}

Received: 27 June 2020 / Accepted: 29 September 2020 / Published online: 7 October 2020

(c) The Author(s) 2020

\begin{abstract}
Background Postoperative morbidity after laparoscopic bariatric surgery is considered higher for patients undergoing revisional versus primary procedures. The objective of this retrospective cohort study was to compare outcomes between patients undergoing primary versus revisional robotically assisted laparoscopic (RAL) Roux-en-Y gastric bypass (RYGB).

Methods Data of all patients who underwent RAL primary and revisional RYGB between 2009 and 2019 at two accredited, high-volume bariatric surgery centers-the Memorial Hermann - Texas Medical Center, Houston, TX, and the Tower Health, Reading Hospital, Reading, PA, were analyzed. Primary outcomes were early ( $<30$ days) and overall postoperative complications. Secondary outcomes included intraoperative complications, operative times, conversions to laparotomy, length of hospital stay, early ( $<30$ days) postoperative readmissions and deaths.

Results Data of 1072 patients were analyzed, including 806 primary and 266 revisional RAL RYGB procedures. Longer operative times (203 versus $154 \mathrm{~min}, P<0.001)$, increased number of readmissions for oral intolerance $(10.5 \%$ versus $6.7 \%, \mathrm{P}=0.046)$ and higher rate of gastrojejunal stricture $(6.4 \%$ versus $2.7 \%, \mathrm{P}=0.013)$ were found in the revisional group. Gastrointestinal leak rates were $0.2 \%$ for the primary versus $1.1 \%$ for the revisional group $(P=0.101)$. Early $(<30$ days $)$ reoperations rates were $2.2 \%$ for the primary versus $1.1 \%$ for the revisional group $(P=0.318)$. There were no statistically significant differences between groups in overall and severe complication rates.

Conclusion Patients undergoing RAL primary and revisional RYGB had comparable overall outcomes, with a non-significant higher early complication rate in the revisional group. Despite the study being underpowered to detect differences in specific complication rates, the morbidity seen in the revisional RYGB group remains markedly below literature reports of revisional laparoscopic RYGB and might suggest a benefit of robotic assistance. Further prospective studies are needed to confirm these results.
\end{abstract}

Keywords Robotic surgery $\cdot$ Gastric bypass $\cdot$ Revisional bariatric surgery

Bariatric surgery is currently established as the most effective treatment for morbid obesity and its related comorbidities [1,2]. The advent of laparoscopy in the early 1990s has

Pouya Iranmanesh

pouya16@hotmail.com

1 Division of Minimally Invasive and Elective General Surgery, Department of Surgery, McGovern Medical School, University of Texas Health Science Center at Houston, 6431 Fannin Street, Houston, TX 77030, USA

2 Weight Loss Surgery and Wellness Center, Tower Health, Reading Hospital, Reading, PA, USA significantly improved perioperative outcomes for patients with obesity and has naturally emerged as the standard approach for most bariatric procedures [3-5]. The overall

\footnotetext{
Texas Laparoscopic Consultants, Houston, TX, USA

Health First Medical Group, Rockledge, FL, USA

5 Michael E. DeBakey Institute for Comparative Cardiovascular Science and Biomedical Devices, Texas A\&M University, College Station, TX, USA
} 
number of bariatric surgeries has constantly been increasing in the past decade across the world, with almost 686,000 procedures performed in 2016, including 51,000 (7.4\%) revisional procedures [6]. The same data show that laparoscopic Roux-en-Y gastric bypass (RYGB) is the second most commonly performed bariatric surgery worldwide after the laparoscopic sleeve gastrectomy (SG), with 191,000 and 341,000 procedures, respectively.

In the US, 42,800 RYGB were performed in 2018, representing $17 \%$ of all bariatric surgeries [7]. Severe early complications after primary laparoscopic RYGB are relatively uncommon nowadays, with anastomotic leak rates ranging between 0.1 and $1.2 \%$ [8-11]. Higher morbidity and mortality have however been described after laparoscopic revisional RYGB, with anastomotic leak rates ranging from 4.5 to $11.8 \%$ [11-19].

Robotically assisted laparoscopic (RAL) surgery offers better visualization, articulated instruments, tremor filtration, ergonomic position and the possibility of performing handsewn anastomoses similarly to open surgery [20,21]. Although little randomized data are available, outcomes after primary RAL bariatric surgery seem to be at least equivalent to the traditional laparoscopic approach. Some larger series have found lower anastomotic leak and stricture rates when using the robotic platform for primary RAL RYGB [22-24]. Smaller studies have suggested the same benefits for patients undergoing revisional RAL RYGB [25-27]. The objective of this study was to compare outcomes between patients undergoing primary versus revisional RAL RYGB. The hypothesis was that both groups would have similar early and late postoperative complication rates.

\section{Material and methods}

\section{Design, setting and participants}

This study was a retrospective analysis of a prospective database containing all patients $>18$ years who underwent RAL primary or revisional RYGB between 2009 and 2019 at two accredited bariatric surgery centers: The University of Texas Health Science Center at Houston and Memorial Hermann - Texas Medical Center (Houston, TX), and Tower Health, Reading Hospital (Reading, PA).

The primary RYGB group included patients who had never undergone any previous bariatric or antireflux surgery. The revisional RYGB group included patients undergoing any of the following surgeries:

- Conversion from any other previous bariatric procedure to RYGB

- Revision of an existing RYGB
- RYGB performed for weight loss purposes in patients who had undergone previous antireflux procedures

Patients undergoing revision of an existing RYGB were included only if at least one anastomosis was revised. Patients undergoing RYGB for purposes other than weight loss (i.e., gastroesophageal reflux control) were excluded.

Surgeons were classified according to their experience in robotic bariatric surgery as senior (> 200 RAL RYGB) and junior ( $<200$ RAL RYGB). All surgeons had performed at least $100 \mathrm{RAL}$ general surgery procedures and 50 primary RAL RYGB and had a minimum of 2 years of bariatric experience beyond their fellowship training.

\section{Technique}

All surgeries were performed using the $\mathrm{Si}$ or Xi version of the da Vinci ${ }^{\circledR}$ Surgical System (Intuitive Incorporation, Sunnyvale, CA, USA). The same surgical technique was used in both institutions. All gastrojejunal anastomoses were performed side-to-side using a fully handsewn technique with absorbable suturing material. A bougie was used to calibrate the gastrojejunal anastomosis to approximately $1.5 \mathrm{~cm}$ final stomal diameter. All jejunojejunal anastomoses were performed in a side-to-side fashion using a $60-\mathrm{mm}$ endoscopic linear stapler and enterotomy defects were closed using a handsewn technique with absorbable suturing material or endoscopic linear stapler as well. Details of our operative technique used for RAL RYGB have been previously published elsewhere [28].

\section{Collected data}

Baseline characteristics included age, gender, body mass index (BMI) at the time of surgery, comorbidities, history of previous abdominal surgeries and length of follow-up after surgery. For the revisional group, types of conversion or revision were also collected. Primary outcomes were early ( $<30$ days after surgery) and overall postoperative complications. Overall postoperative complications included all complications occurring at any time between surgery and end of patient follow-up. Secondary outcomes were intraoperative complications, operative times, conversions to laparotomy, length of hospital stay (LOS), early ( $<30$ days after surgery) readmissions and deaths. Postoperative complications were ranked according to the Dindo-Clavien classification [29], with severe complications defined by a score of $\geq$ IIIa (complications requiring percutaneous, endoscopic or surgical intervention, and/or intensive care unit management). To ensure data completeness and accuracy, electronic records of each patient were reviewed by the primary author between February and August 2019 prior to performing statistical analyses. All readmissions, reoperations, postoperative 
Table 2 Types of revisions

\begin{tabular}{lll}
\hline Initial surgery & $n$ & $\%$ \\
\hline Other bariatric procedures (conversions) & 230 & 86.4 \\
- AGB & 125 & 47.0 \\
- VBG & 44 & 16.5 \\
- SG & 39 & 14.7 \\
- Fixed Molina band & 15 & 5.6 \\
- Transoral gastroplasty & 6 & 2.2 \\
- One-anastomosis gastric bypass & 1 & 0.4 \\
RYGB (revisions) & 24 & 9.0 \\
Antireflux procedures & 10 & 3.8 \\
- Nissen fundoplication & 6 & 2.2 \\
- Hill repair & 2 & 0.8 \\
- Toupet fundoplication & 1 & 0.4 \\
- Transoral incisionless fundoplication & 1 & 0.4 \\
Other & 2 & 0.8 \\
- Lateral gastric plication & 1 & 0.4 \\
- Unclear type of fundoplication & 1 & 0.4 \\
\hline
\end{tabular}

The denominator for all lines is the total cases of revisional procedures $(n=266)$

$A G B$ adjustable gastric band, $V B G$ vertical banded gastroplasty, $S G$ sleeve gastrectomy, $R Y G B$ Roux-en-Y gastric bypass

and overall complication rates on univariate and multivariate regression analyses according to baseline characteristics. Subgroup analyses according to length of follow-up and surgeons experience did not show different results. Detailed complication breakdown can be seen in Table 4 . When analyzing each type of complication separately, the revisional group had significantly higher rates of gastrojejunal stricture and oral intolerance requiring readmission.
All patients readmitted with oral intolerance were treated conservatively with intravenous fluids and antiemetics (grade I). There was no difference between groups for other types of complication.

Severe early ( $<30$ days) postoperative complications (grade $\geq$ IIIa) and their management are shown in Table 5 . The revisional group had a longer mean operative time (203 versus $154 \mathrm{~min}, P<0.001$ ). Rates of readmissions, intraoperative complications, conversions to laparotomy and mean LOS were similar between groups.

Intraoperative complications in the primary group included 2 positive gastrojejunal anastomosis air leak tests (managed with interrupted sutures), 1 misfired stapler load on the gastric remnant (managed by a running suture), 1 liver bleed (managed with electrocautery and hemostatic agents), 1 mesenteric bleed (managed by conversion to laparotomy and vessel ligation) and 1 patient who developed hyperkalemia with $\mathrm{T}$-waves during general anesthesia (managed by insulin and calcium gluconate injections). Intraoperative complications in the revisional group included 3 small bowel injuries (managed by resection and anastomosis) and 1 pneumothorax (managed by pleural drain insertion). Conversion to laparotomy was necessary in three patients in the primary group (due to uncontrolled mesenteric bleeding, intestinal malrotation and small bowel injury, respectively) and one patient in the revisional group (due to small bowel injury). There were no conversions to standard laparoscopy. In the primary group, there was one early death on the first postoperative day due cardiac arrhythmia with asystole and a late death three years after surgery due to an internal hernia with extensive small bowel necrosis and septic shock, who died despite emergency surgery. There were no deaths in the revisional group.
Table 3 Primary and secondary outcome comparison

\begin{tabular}{|c|c|c|c|}
\hline Outcomes & Primary $(n=806)$ & Revisional $(n=266)$ & $P$-value \\
\hline Early (<30 days) complications (\%) & $92(11.4)$ & $42(15.8)$ & 0.069 \\
\hline Early (<30 days) severe complications ${ }^{\mathrm{a}}(\%)$ & $28(3.5)$ & $12(4.5)$ & 0.456 \\
\hline Early (<30 days) reoperations $(\%)$ & $18(2.2)$ & $3(1.1)$ & 0.318 \\
\hline Overall complications $^{\mathrm{b}}(\%)$ & $227(28.2)$ & 87 (32.7) & 0.163 \\
\hline Overall severe complications ${ }^{\mathrm{a}, \mathrm{b}}(\%)$ & $112(13.9)$ & $44(16.5)$ & 0.316 \\
\hline Overall reoperations (\%) & $79(9.8)$ & $25(9.4)$ & 0.905 \\
\hline Readmissions within 30 days (\%) & $67(8.3)$ & $30(11.3)$ & 0.174 \\
\hline Intraoperative complications (\%) & $6(0.6)$ & $4(1.5)$ & 0.237 \\
\hline Operative times, mean (SD), min & $154( \pm 51)$ & $203( \pm 78)$ & $<0.001$ \\
\hline Conversions to laparotomy (\%) & $3(0.4)$ & $1(0.4)$ & 0.999 \\
\hline Length of hospital stay, mean (SD), days & $3.4( \pm 5.1)$ & $3.1( \pm 2.7)$ & 0.866 \\
\hline Deaths $(\%)$ & $2(0.2)$ & 0 & 0.999 \\
\hline
\end{tabular}


Table 4 Complication breakdown

\begin{tabular}{|c|c|c|c|c|c|}
\hline \multirow[t]{2}{*}{ Type of complication } & \multicolumn{2}{|c|}{$\begin{array}{l}\text { Primary } \\
(n=806)\end{array}$} & \multicolumn{2}{|c|}{$\begin{array}{l}\text { Revi- } \\
\text { sional } \\
(n=266)\end{array}$} & \multirow[t]{2}{*}{$P$-value } \\
\hline & $n$ & $\%$ & $n$ & $\%$ & \\
\hline Gastrointestinal leaks & 2 & 0.2 & 3 & 1.1 & 0.101 \\
\hline - Gastrojejunal anastomosis & 1 & 0.1 & 1 & 0.4 & 0.435 \\
\hline - Jejunojejunal anastomosis & 0 & 0 & 1 & 0.4 & 0.248 \\
\hline - Gastric pouch staple line & 0 & 0 & 1 & 0.4 & 0.248 \\
\hline - Gastric remnant staple line & 1 & 0.1 & 0 & 0 & 0.999 \\
\hline Marginal ulcer & 55 & 6.8 & 21 & 7.9 & 0.582 \\
\hline Requiring anastomotic revision & 3 & 0.4 & 1 & 0.4 & 0.999 \\
\hline Gastrojejunal stricture & 22 & 2.7 & 17 & $6.4 \%$ & 0.013 \\
\hline Requiring anastomotic revision & 0 & 0 & 2 & 0.8 & 0.061 \\
\hline Perforated gastrojejunal ulcer & 14 & 1.7 & 1 & 0.4 & 0.134 \\
\hline Perforated jejunojejunal ulcer & 1 & 0.1 & 0 & 0 & 0.999 \\
\hline Oral intolerance requiring readmission & 54 & 6.7 & 28 & 10.5 & 0.046 \\
\hline Internal hernia & 32 & 4.0 & 11 & 4.1 & 0.859 \\
\hline Jejunojejunal obstruction & 5 & 0.6 & 0 & 0 & 0.341 \\
\hline Gastrogastric fistula & 0 & 0 & 1 & 0.4 & 0.248 \\
\hline Small bowel obstruction & 10 & 1.2 & 4 & 1.5 & 0.757 \\
\hline Incisional hernia & 11 & 1.4 & 0 & 0 & 0.075 \\
\hline Esophageal ulcer bleed & 0 & 0 & 1 & 0.4 & 0.248 \\
\hline Unlocalized gastrointestinal bleed & 9 & 1.1 & 0 & 0 & 0.123 \\
\hline Iatrogenic colon injury & 1 & 0.1 & 0 & 0 & 0.999 \\
\hline Ileum necrosis & 0 & 0 & 1 & 0.4 & 0.248 \\
\hline Esophagogastric junction stricture & 0 & 0 & 1 & 0.4 & 0.248 \\
\hline Intraabdominal fluid collection & 1 & 0.1 & 1 & 0.4 & 0.435 \\
\hline Superficial wound infection & 2 & 0.2 & 1 & 0.4 & 0.575 \\
\hline Unexplained, chronic abdominal pain & 40 & 5.0 & 11 & 4.1 & 0.740 \\
\hline Negative diagnostic laparoscopy & 15 & 1.9 & 5 & 1.9 & 0.999 \\
\hline Thromboembolic events & 4 & 0.5 & 2 & 0.8 & 0.642 \\
\hline Pulmonary embolus & 2 & 0.2 & 0 & 0 & 0.999 \\
\hline Deep veinous thrombosis & 2 & 0.2 & 2 & 0.8 & 0.258 \\
\hline Pneumonia & 5 & 0.6 & 1 & 0.4 & 0.999 \\
\hline Atelectasis & 3 & 0.4 & 0 & 0 & 0.999 \\
\hline Myocardial infarction & 1 & 0.1 & 1 & 0.4 & 0.435 \\
\hline Heart failure & 1 & 0.1 & 0 & 0 & 0.999 \\
\hline Nephrolithiasis & 3 & 0.4 & 0 & 0 & 0.999 \\
\hline Urinary tract infection & 10 & 1.2 & 1 & 0.4 & 0.310 \\
\hline Acute renal failure & 0 & 0 & 1 & 0.4 & 0.248 \\
\hline Congestion and fever & 1 & 0.1 & 0 & 0 & 0.999 \\
\hline
\end{tabular}

$P$-values in bold indicate statistically significant results

\section{Discussion}

To the best of our knowledge, this article describes the largest cohort of patients undergoing revisional RAL RYGB in the literature $(n=266)$. In this study, patients undergoing RAL primary and revisional RYGB had similar outcomes in terms of overall postoperative complication rates. The incidences of specific complications such as leaks, marginal ulcer or internal hernia were similar between groups, except for a higher rate of gastrojejunal strictures and readmissions for oral intolerance among the revisional group.

Differences between groups in baseline characteristics were most likely due to patients undergoing revisional surgery at least a few years after the initial procedure. At that time, patients usually had a lower BMI and varying degree of comorbidities remission, especially if they underwent surgery for complications such as persistent nausea or dysphagia. The higher prevalence of preoperative GERD in the revisional group could be explained by the natural postoperative history of procedures such as vertical banded gastroplasty, gastric band placement and sleeve gastrectomy, which have all been shown to cause de novo or worsening of GERD postoperatively in some patients [30-32] and for whom RYGB provides excellent results in terms of postoperative reflux control $[32,33]$. Although statistically significant, the small mean difference in lengths of follow-up between groups ( 0.7 months) is unlikely to have clinical significance. Univariate and multivariate regression analyses were performed to adjust for these differences, without modifying the initial results.

Gastrointestinal leaks are a dreaded complication in bariatric surgery. Previous studies have suggested a lower leak rate after primary RAL RYGB compared to laparoscopic RYGB [22-24]. When considering all types of leaks, the incidence was only $0.2 \%$ (2/806) for the primary group and $1.1 \%(3 / 266)$ for the revisional group $(P=0.101)$. Among them, gastrojejunal anastomotic leaks rates were $0.1 \%$ $(1 / 806)$ and $0.4 \%(1 / 266)$, respectively $(P=0.435)$. Leak rates in the literature range from $0.1 \%$ to $1.2 \%$ after primary laparoscopic RYGB [8-11] and 4.5 to $11.8 \%$ after revisional laparoscopic RYGB [11-19]. While the gastrointestinal leak rate found after primary RAL RYGB in this study $(2 / 806$, $0.2 \%$ ) was consistent with the lowest leak rates found in the literature, the incidence of leaks after revisional RYGB (3/266, 1.1\%) was markedly below what has been reported with the laparoscopic approach, suggesting an advantage of robotic assistance for revisional RYGB. Potential explanations for this difference are better visualization, increased mobility provided by articulated instruments and a more ergonomic position at the robotic console, possibly leading to a more precise dissection, better tissue handling and less surgeon fatigue during these procedures where extensive lysis of adhesions is often required. This more precise dissection could lead to improved maintenance of blood supply to tissues in an often hostile reoperative field. The possibility to perform fully handsewn anastomoses comparable to open surgery could be another factor, which has been analyzed for primary RYGB in other studies [22, 24]. Surgeon's experience with robotically assisted bariatric surgery certainly 
Table 5 Early severe postoperative complication breakdown and management

\begin{tabular}{|c|c|c|c|}
\hline Type of complication & $n$ & Grade & Management \\
\hline \multicolumn{4}{|l|}{ Primary group } \\
\hline Gastrojejunal anastomosis leak & 1 & IV & Laparoscopic washout and drainage, ICU management \\
\hline Gastric remnant staple line leak & 1 & IIIb & $\begin{array}{l}\text { Laparoscopic washout and drainage, } \\
\text { gastrostomy tube insertion }\end{array}$ \\
\hline Bleeding marginal ulcer & 1 & IIIa & Endoscopic hemostasis, PPI and sucralfate \\
\hline Gastrojejunal anastomosis stricture & 4 & IIIa & Endoscopic balloon dilatation \\
\hline Jejunojejunal anastomosis obstruction & 5 & IIIb & Laparoscopic jejunojejunal anastomosis revision \\
\hline Incarcerated incisional hernia & 4 & $\mathrm{IIIb}$ & Laparoscopic or open incisional hernia repair \\
\hline Internal hernia & 2 & IIIb & Laparoscopic reduction and closure of defects \\
\hline Small bowel obstruction (early adhesions) & 4 & IIIb & Laparoscopic lysis of adhesions \\
\hline Iatrogenic colon perforation & 1 & IV & Open colon resection, ICU management \\
\hline Intraabdominal fluid collection & 1 & IIIa & Interventional radiology drain insertion \\
\hline Large ureteral lithiasis & 1 & IIIb & Lithotripsy under general anesthesia \\
\hline Heart failure & 1 & IV & ICU management \\
\hline Myocardial infarction & 1 & IV & ICU management and PTCA \\
\hline Bilateral pulmonary emboli & 1 & IIIa & Percutaneous catheter thrombolysis and anticoagulation \\
\hline \multicolumn{4}{|l|}{ Revisional group } \\
\hline Gastrojejunal anastomosis leak & 1 & IIIb & Laparoscopic washout and drainage \\
\hline Jejunojejunal anastomosis leak & 1 & IIIa & Interventional radiology drain insertion \\
\hline Gastric pouch staple line leak & 1 & IIIa & Interventional radiology drain insertion \\
\hline Gastrojejunal anastomosis stricture & 4 & IIIa & Endoscopic balloon dilatation \\
\hline Internal hernia & 1 & IIIb & Laparoscopic small bowel resection and closure of defects \\
\hline Small bowel obstruction (early adhesions) & 1 & IIIb & Laparoscopic lysis of adhesions \\
\hline Bleeding esophageal ulcer & 1 & IIIa & Endoscopic hemostasis and PPI \\
\hline Myocardial infarction & 1 & IV & ICU management and PTCA \\
\hline Aspiration pneumonia & 1 & IV & ICU management with mechanical ventilation and antibiotics \\
\hline
\end{tabular}

Early is defined by $<30$ days after surgery. Complications are graded according to the Dindo-Clavien classification. Severe complications are defined by a grade $\geq$ IIIa

ICU intensive care unit, PPI proton pumb inhibitors, PTCA percutaneous transluminal coronary angioplasty

played a role as well. Buchs et al. found that only 14 cases were necessary to overcome the learning curve for RAL RYGB [34]. In their systematic review, Pernar et al. found that less than 100 cases were sufficient to achieve plateau performance for most general surgery subspecialties [35]. All surgeons involved in this study had an experience of at least 150 RAL procedures, but there was no difference in outcomes between senior and junior surgeons.

When analyzing each type of complication separately, the only statistically significant differences were higher rates of readmissions for oral intolerance and gastrojejunal strictures in the revisional group. Oral intolerance was a minor complication in all patients, requiring only IV fluids and antiemetics (grade I). Higher rates of marginal ulcer and gastrojejunal stricture have been described previously after revisional RYGB [16]. Possible mechanisms include decreased micro-vascularization in tissue that has had previous surgery, which may lead to an exaggerated scar response. Macro-vascularization, i.e., left gastric artery perfusion, appears to be preserved, as evidenced by near equivalent leak rates in the revisional group. In this study, all gastrojejunal strictures were successfully treated by endoscopic balloon dilatation in both groups (grade IIIa), except for $2 / 17$ patients from the revisional group who required surgical management (grade IIIb). This trend towards higher rate of surgical revisions for gastrojejunal stricture in patients undergoing revisional RYGB was however not significant. Of note, there was no difference between groups in rates of marginal ulcers and number of patients requiring gastrojejunal anastomosis revision due to refractory marginal ulcer.

The relatively high number of overall postoperative complications is most likely due to the thorough tracking of all postoperative adverse events, even minor complications. Moreover, complications were collected as far as 10 years after surgery for patients with longer follow-ups. The rates of severe complications, including early ( $<30$ days) severe 
complications and reoperations, were however comparable to previously published studies [16, 36, 37].

In this study, conversion to laparotomy was necessary in only $0.4 \%$ of patients in both groups (3/806 and 1/266, respectively). This rate is especially low for patients undergoing revisional RYGB compared to previous reports of conversion rates as high as $14 \%[27,28]$.

This study has several limitations. It is a retrospective data analysis and not a randomized or prospective study. To reduce reporting biases, all patient records were however manually verified, including endoscopic, radiological and surgical reports. Even though no statistical difference was found between groups in terms of gastrointestinal leak rates $(0.2 \%$ vs $1.1 \%, P=0.101)$, this study was underpowered to reject the null hypothesis for specific complications, potentially leading to a type II error. As expected, baseline characteristics between groups were markedly different, which could have resulted in a selection bias. To minimize this potential bias, univariate and multivariate regression analyses were performed to assess confounding factors among baseline characteristics and found no difference in outcomes. The asymmetrical distribution of cases between the two institutions (58\% of primary and $89 \%$ of revisional cases were performed in one site) could have led to a selection bias as well. Another limitation is the relatively low percentage of patients with follow-up greater than 12 months (approximately $50 \%$ in each group). Low postoperative follow-up percentages have been previously reported in the bariatric population [38], and this study is unfortunately no exception. Since the primary outcome of this study was postoperative complications and given a follow-up of at least 3 months for all patients, the authors believe that only late postoperative complications such as marginal ulcer or internal hernia could have potentially been missed for some patients. This possibility should not significantly change the main conclusions of this article. Ideally, a randomized clinical study should be performed to assess a potential advantage of robotically assisted versus laparoscopic surgery in patients undergoing revisional RYGB. The feasibility of such a study is however very limited due to the difficulty of developing equivalent expertise in robotically assisted and laparoscopic revisional bariatric surgery in a single center.

In conclusion, this study showed similar overall early and late complication rates between primary and revisional RAL RYGB, with higher rates of gastrojejunal strictures and readmissions for oral intolerance in the revisional group. Despite the study being underpowered to detect a statistically significant difference between groups in specific complications such as gastrointestinal leak rates, these results suggest that the higher morbidity reported in the literature in patients undergoing laparoscopic revisional RYGB can potentially be decreased, if not brought down, to the low morbidity seen in primary procedures, when using the robotic approach.
Further prospective studies are needed to confirm these results.

Funding: Open access funding provided by University of Geneva.

\section{Compliance with ethical standards}

Disclosures This study did not receive any funding. Individual disclosures are as follow: Dr. L. Katz is a consultant for Boehringer labs (not linked to this study), Dr. S. Myers is a consultant for Ethicon (not linked to this study), Dr. B. Snyder receives teaching honoraria from Intuitive Surgical, Dr. S. Shah is the recipient of a research grant from Intuitive Surgical (not used for this study) and is a consultant for Hatchleaf and C-SATS (not linked to this study) and Dr. E. Wilson receives teaching Honoraria from Intuitive Surgical. Drs. P. Iranmanesh, J. Fam, T. Nguyen, D. Talarico, K. Chandwani, K. Bajwa, M. Felinski, S. Mehta, P. Walker, T. Wilson, A. Rivera and C. Klein have no conflicts of interest or financial ties to disclose.

Open Access This article is licensed under a Creative Commons Attribution 4.0 International License, which permits use, sharing, adaptation, distribution and reproduction in any medium or format, as long as you give appropriate credit to the original author(s) and the source, provide a link to the Creative Commons licence, and indicate if changes were made. The images or other third party material in this article are included in the article's Creative Commons licence, unless indicated otherwise in a credit line to the material. If material is not included in the article's Creative Commons licence and your intended use is not permitted by statutory regulation or exceeds the permitted use, you will need to obtain permission directly from the copyright holder. To view a copy of this licence, visit http://creativecommons.org/licenses/by/4.0/.

\section{References}

1. Buchwald H; Consensus Conference Panel (2005) Consensus conference statement bariatric surgery for morbid obesity: health implications for patients, health professionals, and third-party payers. Surg Obes Relat Dis 1(3):371-381

2. Sjöström L, Narbro K, Sjöström CD, Karason K, Larsson B, Wedel $\mathrm{H}$ et al (2007) Effects of bariatric surgery on mortality in Swedish obese subjects. N Engl J Med 357(8):741-752

3. Favretti F, Cadière GB, Segato G, Bruyns G, De March F, Himpens J et al (1995) Laparoscopic Placement of Adjustable Silicone Gastric Banding: Early Experience. Obes Surg 5(1):71-73

4. DeMaria EJ, Sugerman HJ, Kellum JM, Meador JG, Wolfe LG (2002) Results of 281 consecutive total laparoscopic Roux-en-Y gastric bypasses to treat morbid obesity. Ann Surg 235(5):640-645

5. Luján JA, Frutos MD, Hernández Q, Liron R, Cuenca JR, Valero $\mathrm{G}$ et al (2004) Laparoscopic versus open gastric bypass in the treatment of morbid obesity: a randomized prospective study. Ann Surg 239(4):433-437

6. Angrisani L, Santonicola A, Iovino P, Vitiello A, Higa K, Himpens $\mathrm{J}$ et al (2018) IFSO Worldwide Survey 2016: primary, endoluminal, and revisional procedures. Obes Surg 28(12):3783-3794

7. English WJ, DeMaria EJ, Hutter MM, Kothari SN, Mattar SG, Brethauer S et al (2020) American Society for Metabolic and Bariatric Surgery 2018 estimate of metabolic and bariatric procedures performed in the United States. Surg Obes Relat Dis 16(4):457-463 
8. Hutter MM, Schirmer BD, Jones DB, Ko CY, Cohen ME, Merkow RP et al (2011) First report from the American College of Surgeons Bariatric Surgery Center Network: laparoscopic sleeve gastrectomy has morbidity and effectiveness positioned between the band and the bypass. Ann Surg 254(3):410-420

9. Carrasquilla C, English WJ, Esposito P, Gianos J (2004) Total stapled, total intra-abdominal (TSTI) laparoscopic Roux-en-Y gastric bypass: one leak in 1000 cases. Obes Surg 14(5):613-617

10. Alizadeh RF, Li S, Inaba C, Penalosa P, Hinojosa MW, Smith BR et al (2018) Risk factors for gastrointestinal leak after bariatric surgery: MBASQIP analysis. J Am Coll Surg 227(1):135-141

11. Smith MD, Adeniji A, Wahed AS, Patterson E, Chapman W, Courcoulas AP et al (2015) Technical factors associated with anastomotic leak after Roux-en-Y gastric bypass. Surg Obes Relat Dis 11(2):313-320

12. Pędziwiatr M, Małczak P, Wierdak M, Rubinkiewicz M, Pisarska M, Major P et al (2018) Revisional gastric bypass is inferior to primary gastric bypass in terms of short- and long-term outcomessystematic review and meta-analysis. Obes Surg 28(7):2083-2091

13. Lee S, Carmody B, Wolfe L, Demaria E, Kellum JM, Sugerman $\mathrm{H}$ et al (2007) Effect of location and speed of diagnosis on anastomotic leak outcomes in 3828 gastric bypass cases. J Gastrointest Surg 11(6):708-713

14. Gray KD, Moore MD, Elmously A, Bellorin O, Zarnegar R, Dakin G (2018) Perioperative outcomes of laparoscopic and robotic revisional bariatric surgery in a complex patient population. Obes Surg 28(7):1852-1859

15. Amiki M, Seki Y, Kasama K, Hashimoto K, Kitagawa M, Umezawa A et al (2020) Revisional bariatric surgery for insufficient weight loss and gastroesophageal reflux disease: our 12-year experience. Obes Surg 30(5):1671-1678

16. Axer S, Szabo E, Agerskov S, Näslund I (2019) Predictive factors of complications in revisional gastric bypass surgery: results from the Scandinavian Obesity Surgery Registry. Surg Obes Relat Dis 15(12):2094-2100

17. Zhang L, Tan WH, Chang R, Eagon JC (2015) Perioperative risk and complications of revisional bariatric surgery compared to primary Roux-en-Y gastric bypass. Surg Endosc 29(6):1316-1320

18. Radtka JF 3rd, Puleo FJ, Wang L, Cooney RN (2010) Revisional bariatric surgery: who, what, where, and when? Surg Obes Relat Dis 6(6):635-642

19. Mor A, Keenan E, Portenier D, Torquati A (2013) Case-matched analysis comparing outcomes of revisional versus primary laparoscopic Roux-en-Y gastric bypass. Surg Endosc 27(2):548-552

20. Corcione F, Esposito C, Cuccurullo D, Settembre A, Miranda $\mathrm{N}$, Amato F et al (2005) Advantages and limits of robot-assisted laparoscopic surgery: preliminary experience. Surg Endosc 19(1):117-119

21. Van Koughnett JA, Jayaraman S, Eagleson R, Quan D, van Wynsberghe A, Schlachta CM (2009) Are there advantages to roboticassisted surgery over laparoscopy from the surgeon's perspective? J Robot Surg 3(2):79-82

22. Snyder BE, Wilson T, Leong BY, Klein C, Wilson EB (2010) Robotic-assisted Roux-en-Y Gastric bypass: minimizing morbidity and mortality. Obes Surg 20(3):265-270

23. Snyder BE, Wilson T, Scarborough T, Yu S, Wilson EB (2008) Lowering gastrointestinal leak rates: a comparative analysis of robotic and laparoscopic gastric bypass. J Robot 2(3):159-163

24. Buchs NC, Morel P, Azagury DE, Jung M, Chassot G, Huber $\mathrm{O}$ et al (2014) Laparoscopic versus robotic Roux-en-Y gastric bypass: lessons and long-term follow-up learned from a large prospective monocentric study. Obes Surg 24(12):2031-2039

25. Clapp B, Liggett E, Jones R, Lodeiro C, Dodoo C, Tyroch A (2019) Comparison of robotic revisional weight loss surgery and laparoscopic revisional weight loss surgery using the MBSAQIP database. Surg Obes Relat Dis 15(6):909-919

26. Snyder B, Wilson T, Woodruff V, Wilson E (2013) Robotically assisted revision of bariatric surgeries is safe and effective to achieve further weight loss. World J Surg 37(11):2569-2573

27. Buchs NC, Pugin F, Azagury DE, Huber O, Chassot G, Morel P (2014) Robotic revisional bariatric surgery: a comparative study with laparoscopic and open surgery. Int J Med Robot 10(2):213-217

28. Shah SK, Walker PA, Snyder BE, Wilson EB (2015) Essentials and future directions of robotic bariatric surgery. In: Kroh M, Chalikonda S (eds) Essentials of robotic surgery. Springer, Cham, pp 73-80

29. Dindo D, Demartines N, Clavien PA (2004) Classification of surgical complications: a new proposal with evaluation in a cohort of 6336 patients and results of a survey. Ann Surg 240(2):205-213

30. Howard DD, Caban AM, Cendan JC, Ben-David K (2011) Gastroesophageal reflux after sleeve gastrectomy in morbidly obese patients. Surg Obes Relat Dis 7(6):709-713

31. de Jong JR, Besselink MG, Van Ramshorst B, Gooszen HG, Smout AJ (2010) Effects of adjustable gastric banding on gastroesophageal reflux and esophageal motility: a systematic review. Obes Rev 11(4):297-305

32. Ortega J, Escudero MD, Mora F, Sala C, Flor B, Marinez-Valls J et al (2004) Outcome of esophageal function and 24-hour esophageal $\mathrm{pH}$ monitoring after vertical banded gastroplasty and Rouxen-Y gastric bypass. Obes Surg 14(8):1086-1094

33. Madalosso CA, Gurski RR, Callegari-Jacques SM, Navarini D, Mazzini G, Pereira Mda S (2016) The Impact of Gastric Bypass on Gastroesophageal Reflux Disease in Morbidly Obese Patients. Ann Surg 263(1):110-116

34. Buchs NC, Pugin F, Bucher P, Hagen ME, Chassot G, KoutnyFong P et al (2012) Learning curve for robot-assisted Roux-en-Y gastric bypass. Surg Endosc 26(4):1116-1121

35. Pernar LIM, Robertson FC, Tavakkoli A, Sheu EG, Brooks DC, Smink DS (2017) An appraisal of the learning curve in robotic general surgery. Surg Endosc 31(11):4583-4596

36. Chang SH, Stoll CR, Song J, Varela JE, Eagon CJ, Colditz GA (2014) The effectiveness and risks of bariatric surgery: an updated systematic review and meta-analysis, 2003-2012. JAMA Surg 149(3):275-287

37. Birkmeyer NJ, Dimick JB, Share D, Hawasli A, English WJ, Genaw J et al (2010) Hospital complication rates with bariatric surgery in Michigan. JAMA 304(4):435-442

38. Poole NA, Al Atar A, Kuhanendran D, Bidlake L, Fiennes A, McCluskey S et al (2005) Compliance with surgical after-care following bariatric surgery for morbid obesity: a retrospective study. Obes Surg 15(2):261-265

Publisher's Note Springer Nature remains neutral with regard to jurisdictional claims in published maps and institutional affiliations. 